\title{
A SPARSE DISCRETIZATION FOR INTEGRAL EQUATION FORMULATIONS OF HIGH FREQUENCY SCATTERING PROBLEMS *
}

\author{
DAAN HUYBRECHS ${ }^{\dagger}$ AND STEFAN VANDEWALLE ${ }^{\dagger}$
}

\begin{abstract}
We consider two-dimensional scattering problems, formulated as an integral equation defined on the boundary of the scattering obstacle. The oscillatory nature of high-frequency scattering problems necessitates a large number of unknowns in classical boundary element methods. In addition, the corresponding discretization matrix of the integral equation is dense. We formulate a boundary element method with basis functions that incorporate the asymptotic behavior of the solution at high frequencies. The method exhibits the effectiveness of asymptotic methods at high frequencies with only few unknowns, but retains accuracy for lower frequencies. New in our approach is that we combine this hybrid method with very effective quadrature rules for oscillatory integrals. As a result, we obtain a sparse discretization matrix for the oscillatory problem. Moreover, numerical experiments indicate that the accuracy of the solution actually increases with increasing frequency. The sparse discretization applies to problems where the phase of the solution can be predicted a priori, for example in the case of smooth and convex scatterers.
\end{abstract}

Key words. integral equations, high-frequency scattering, oscillatory integrals, steepest descent

AMS subject classifications. 45B05, 65D30, 41A60

DOI. $10.1137 / 060651525$

1. Introduction. The accurate numerical modeling of physical problems involving strong oscillations is a challenging problem. Scattering problems in unbounded domains are often modeled by an integral equation defined on the boundary of the scattering obstacle. As such, the problem on an unbounded domain is reduced to a lower-dimensional problem on a bounded domain. Numerically, these are two important advantages. Still, the method has its drawbacks, and difficulties arise as the frequency of the problem increases. Contrary to the case for partial differential equations, the discretization matrix of an integral equation is dense. Furthermore, in order to represent an oscillatory solution, the number of unknowns in a boundary element approach has to be large. Typically, one chooses a fixed number of unknowns per wavelength per dimension. This results in a linear system with a very large and dense discretization matrix. Hence, classical solution methods for scattering problems rapidly become prohibitively expensive [4].

Substantial efforts have been made over the last two decades to overcome these difficulties. One direction has been to improve on the solution time required to solve the dense linear system. The fast multipole method achieves a fast matrix-vector product in $O(N \log N)$ operations, when the number of unknowns $N$ increases at least linearly with the wavenumber [30]. The fast matrix-vector product can be combined with a preconditioned iterative technique for an efficient overall solution algorithm [10]. Another direction is given by a number of asymptotic methods, such as geometrical optics, physical optics, and the geometrical theory of diffraction [17, 23]. A common

\footnotetext{
* Received by the editors February 4, 2006; accepted for publication (in revised form) March 15, 2007; published electronically October 5, 2007.

http://www.siam.org/journals/sisc/29-6/65152.html

${ }^{\dagger}$ Department of Computer Science, Katholieke Universiteit Leuven, Celestijnenlaan 200A, B-3001 Leuven, Belgium (daan.huybrechs@cs.kuleuven.be, stefan.vandewalle@cs.kuleuven.be). The first author was supported by the Institute for the Promotion of Innovation through Science and Technology in Flanders (IWT-Vlaanderen).
} 
characteristic of asymptotic methods is that they have an error of the order $O\left(k^{-n}\right)$, where $k$ is the wavenumber and where the exponent $n$ is typically equal to 1 or 2 . This means that the accuracy of such methods improves with increasing frequencies. Asymptotic methods break down for low to moderate frequencies, however.

A more recent trend is the combination of finite element methods with asymptotic methods. This is achieved by considering basis functions that are, e.g., piecewise polynomial, multiplied by the asymptotic form of the solution at large frequencies. The asymptotic behavior of the solution to the problem of scattering by smooth convex obstacles was analyzed in [27]. Motivated by these results, a hybrid scheme was considered in [1]. The authors report an overall solution method that requires $O\left(k^{1 / 3}\right)$ operations as a function of the wavenumber, a huge improvement over the linear dependence on $k$. The basis functions are piecewise polynomials, multiplied by plane waves in a number of directions. Similar hybrid methods with even better results are proposed in $[2,7,25,24,15,12]$. A number of operations that is independent of the wavenumber, for a fixed error, is achieved by Bruno et al. in [7] for the scattering by smooth convex obstacles, and by Langdon and Chandler-Wilde in [25] for scattering on a half-plane. In the present paper, we combine a similar approach with recent insights into the behavior of oscillatory integrals [21, 19]. As a result, we obtain a small and highly sparse discretization matrix. In addition, the accuracy of the solution actually increases with increasing frequency.

We start the paper in section 2 with a brief review of a suitable quadrature rule for the evaluation of oscillatory integrals that have the general form

$$
I[f]:=\int_{a}^{b} f(x) e^{i k g(x)} \mathrm{d} x,
$$

where both $f$ and $g$ are smooth functions and where the wavenumber $k$ determines the frequency of the oscillations. The review is based on results by Iserles and Nørsett in [21] and by the authors in [19]. We show how Filon-type quadrature rules using derivatives can be constructed that have the form

$$
I[f] \approx Q[f]:=\sum_{l=0}^{n} \sum_{j=1}^{d_{l}} w_{l, j} f^{(j)}\left(x_{l}\right)
$$

such that the accuracy of the rule improves with increasing frequency. This is quite contrary to the rapid deterioration of classical quadrature rules, based on polynomial interpolation, for increasingly oscillatory integrals. The asymptotic order of accuracy as a function of the frequency is $O\left(k^{-s-1}\right)$, where the value of $s$ depends on the number of derivatives used. This is much like the behavior of truncated asymptotic expansions. However, due to the nature of the constructed rules, the result is exact for an arbitrarily large family of functions regardless of the frequency. The main difference with asymptotic expansions therefore is that there is no breakdown at low frequencies. In general, one can also expect a smaller error for moderate frequencies.

The results of [19] are generalized in section 3 to an appropriately chosen model form

$$
I_{H}[f]:=\int_{a}^{b} f(x) H_{\nu}^{(1)}\left(k g_{1}(x)\right) e^{i k g_{2}(x)} \mathrm{d} x
$$

where $f, g_{1}$, and $g_{2}$ are assumed to be smooth functions. The function $H_{\nu}^{(1)}(z)$ is the Hankel function of the first kind and order $\nu$; it is an oscillatory function for large 
arguments. The model form is chosen to represent the integrals that will appear later in the solution of scattering problems. The resulting quadrature rule has the same form as (1.2). The scattering problem is introduced in section 4. The quadrature rules are subsequently used in the discretization of the oscillatory integral equation in section 5. We identify a setting in which each row of the discretization matrix corresponds to the discretization of a specific one-dimensional oscillatory integral with a known phase. Owing to the small number of quadrature points required for the evaluation of such integrals, the discretization matrix is sparse. The accuracy of the solution improves because the quadrature rules themselves improve for increasing frequencies.

We illustrate the method with numerical results in section 6 . We consider the scattering of a plane wave and of a circular wave, by a circle and by an ellipse, respectively. We end the paper with some concluding remarks in section 7 .

2. The efficient evaluation of oscillatory integrals. The classical approach for the evaluation of oscillatory integrals is to use a fixed number of quadrature points per wavelength. This automatically leads to a number of operations that scales linearly with the frequency. However, the asymptotic expansion of an oscillatory integral for large frequencies reveals that the value of the integral is actually determined by the behavior of the integrand near a small set of special points $[31,5]$. These are the boundary points of the interval, and the so-called stationary points.

Consider the oscillatory integral (1.1), where both $f$ and $g$ are smooth functions. We call $f$ the amplitude, and $g$ the oscillator, of the integral. The stationary points of (1.1) are all solutions $\xi$ to the equation $g^{\prime}(\xi)=0, \xi \in[a, b]$. A stationary point is said to have order $r$ if $g^{(j)}(\xi)=0, j=1, \ldots, r$, but $g^{(r+1)}(\xi) \neq 0$. The importance of such points lies in the fact that, locally, the integrand does not oscillate near a stationary point. Away from all stationary points and the boundary points $a$ and $b$, the oscillations of the integrand increasingly cancel out. Hence, such regions do not contribute much to the value of the integral.

Mathematically, this property is reflected in the asymptotic expansion of (1.1). It can be shown that integral (1.1) with one interior stationary point of order $r$ has an asymptotic expansion of the form (see [31])

$$
I[f] \sim \sum_{j=0}^{\infty} \frac{c_{j}[f]}{k^{(j+1) /(r+1)}}, \quad k \rightarrow \infty .
$$

It is proved in [21] that the first few coefficients $c_{j}[f]$ of the asymptotic expansion depend only on the first few derivatives of $f$ and $g$, evaluated at the boundary points and at the stationary points. A number of recent methods exploit this behavior in order to obtain an approximation of $I[f]$ that improves with increasing $k[21,19,26$, 29]. We recall one particularly useful approach, which leads to a quadrature rule with a classical form. We refer the reader to [20] for a more general overview.

2.1. A numerical steepest descent method. A well-known technique for obtaining the asymptotic expansion (2.1) is the method of steepest descent [5,33]. Justified by Cauchy's integral theorem for analytic functions, the integration path is deformed into a complex integration path that has more desirable properties. Specifically, the new path is chosen such that the integrand is not oscillatory along the path and has exponential decay. The asymptotic expansion can then be derived from the resulting line integrals in the complex plane. Asymptotic expansions have the disadvantage, however, that the error is not controllable for low values of $k$. Rather than 
forming the asymptotic expansion, the line integrals can also be evaluated numerically. This numerical approach leads to a method that improves with increasing $k$, but that retains accuracy for small values of $k$ [19].

Assume that both $f$ and $g$ are analytic functions. Subdivide the integration interval $[a, b]$ into subintervals $\left[a_{l}, b_{l}\right], l=1, \ldots, L$, such that $g^{\prime}(x) \neq 0, x \in\left(a_{l}, b_{l}\right)$, i.e., the oscillator $g$ is a monotonic function on $\left[a_{l}, b_{l}\right]$. Then the inverse of $g$ exists uniquely on $\left[a_{l}, b_{l}\right]$, and it is also analytic. We denote it by $g_{l}^{-1}$. The integral over $\left[a_{l}, b_{l}\right]$ is evaluated by deforming the integration path into the complex plane. Define the path $h_{x, l}(p)$ by

$$
h_{x, l}(p)=g_{l}^{-1}(g(x)+i p), \quad x \in\left[a_{l}, b_{l}\right] .
$$

This path is called the path of steepest descent. It follows immediately from the definition that $e^{i k g\left(h_{x, l}(p)\right)}=e^{-k p} e^{i k g(x)}$. Hence, the line integral along the new integration path, originating in the point $x$ and terminating in the point $h_{x, l}(P)$, can be written as

$$
S_{l}[f ; x]=e^{i k g(x)} \int_{0}^{P} f\left(h_{x, l}(p)\right) h_{x, l}^{\prime}(p) e^{-k p} \mathrm{~d} p, \quad P>0 .
$$

The integrand of (2.3) is not oscillatory and decays exponentially fast. The integral over $\left[a_{l}, b_{l}\right]$ can now be approximated by $S_{l}\left[f ; a_{l}\right]-S_{l}\left[f ; b_{l}\right]$. In general, the value of $I[f]$ can be approximated by a sum of nonoscillatory line integrals with exponentially small error,

$$
I[f]=\sum_{l=1}^{L}\left(S_{l}\left[f ; a_{l}\right]-S_{l}\left[f ; b_{l}\right]\right)+O\left(e^{-k P}\right),
$$

with $P>0$. The larger $P$, the better the approximation, but for all finite $P$ the error decays exponentially fast as the frequency parameter $k$ increases. In many cases of practical interest, the limit case $P \rightarrow \infty$ is possible, and the error $O\left(e^{-k P}\right)$ vanishes. In that case, decomposition (2.4) is exact.

2.2. A quadrature rule for oscillatory integrals. The decomposition (2.4) can be used to obtain a quadrature rule for $I[f]$ with a classical form. Approximating $f$ by its truncated Taylor series at each point $a_{l}$ and $b_{l}$, we find a quadrature rule using derivatives of the form

$$
Q[f]:=\sum_{l=0}^{L} \sum_{j=0}^{d_{l}} w_{l, j} f^{(j)}\left(x_{l}\right),
$$

with $x_{0}=a, x_{l}=b_{l}, l=1, \ldots, L$, and with the weights given by

$$
\begin{aligned}
& w_{0, j}=S_{1}\left[\frac{(x-a)^{j}}{j !} ; a\right] \\
& w_{l, j}=-S_{l}\left[\frac{\left(x-x_{l}\right)^{j}}{j !} ; x_{l}\right]+S_{l+1}\left[\frac{\left(x-x_{l}\right)^{j}}{j !} ; x_{l}\right], \quad l=1, \ldots, L-1, \\
& w_{l, j}=-S_{l}\left[\frac{(x-b)^{j}}{j !} ; b\right] .
\end{aligned}
$$

Note that this rule uses only local information of $f$ : its values and derivatives at the endpoints $a$ and $b$ of the integration region and at all the stationary points in 
between. Yet the approximation $I[f] \approx Q[f]$ is very accurate and becomes increasingly accurate with increasing values of the frequency parameter $k$. It can be shown that the first few terms of the asymptotic expansion of the error $I[f]-Q[f]$ vanish. This is due to the fact that these terms depend only on the derivatives of $f$ at exactly the points we consider in the quadrature rule. Hence, the approximation has a high asymptotic accuracy. The exact order depends on the number of derivatives $d_{l}$ that are used and on the order of the stationary points involved [19].

3. Specialized quadrature rules. The quadrature rule of the previous section applies only to the model integral (1.1). Intuitively, however, one sees that the ideas can be readily generalized to any oscillatory integral. The value of an oscillatory integral is determined by the behavior of the integrand near the endpoints of the integration interval, and near the points where the integrand locally does not oscillate. In order to construct similar quadrature rules, one requires knowledge of the phase of the integral. In this section, we will construct such rules for a family of integrals that will arise in the scattering problem discussed later. In particular, the integrand involves an oscillatory Hankel function.

3.1. A generalized model form. Consider the oscillatory integral

$$
I_{H}[f]=\int_{a}^{b} f(x) H_{\nu}^{(1)}\left(k g_{1}(x)\right) e^{i k g_{2}(x)} \mathrm{d} x,
$$

where $f, g_{1}$, and $g_{2}$ are smooth functions and $H_{\nu}^{(1)}(z)$ is the Hankel function of the first kind of order $\nu$. The Hankel function of order zero, $H_{0}^{(1)}(z)$, has a logarithmic singularity at $z=0$. Hankel functions of higher order have algebraic singularities of the form $1 / z^{\nu}, z \rightarrow 0[3]$.

For large arguments, the Hankel functions behave like an oscillatory complex exponential with a decaying amplitude,

$$
H_{\nu}^{(1)}(z) \sim \sqrt{\frac{2}{\pi z}} e^{i\left(z-\frac{1}{2} \nu \pi-1 / 4 \pi\right)}, \quad-\pi<\arg z<\pi, \quad|z| \rightarrow \infty .
$$

Hence, the oscillator of the integrand of (3.1) is approximately given by

$$
g(x)=g_{1}(x)+g_{2}(x),
$$

up to the addition of a constant. The Hankel function decays exponentially fast for complex arguments with a positive imaginary part, as can be seen from the asymptotic behavior (3.2). This means that the approach of section 2.2 using the path of steepest descent is applicable. Hence, we conjecture that a quadrature rule exists of the form

$$
I_{H}[f] \approx Q_{H}[f]:=\sum_{l=0}^{L} \sum_{j=0}^{d_{l}} w_{l, j}^{H} f^{(j)}\left(x_{l}\right) .
$$

In the remainder of the section, we will prove this conjecture, determine the quadrature abscissae $x_{l}$, and show how the weights can be computed efficiently.

3.2. Construction of the quadrature rule. We start by stating some assumptions on the functions $f$ and $g$, which are needed to guarantee the integrability and analyticity of the integrand in (3.1). First, we assume that $f$ is analytic in an open complex neighborhood $D$ of $[a, b]$, so that $[a, b] \subset$ int $D$. Likewise, we assume 
that $g_{1}$ and $g_{2}$ are nonsingular and analytic in $D$, except possibly along a branch cut that extends from $a$ or $b$ to the boundary of the region $D$; i.e., $a$ and $b$ may be branch points but not singular points. We assume furthermore that $g(x)$, defined by (3.3), is strictly monotonic on the open interval $(a, b)$ and hence invertible, but possibly $g^{\prime}(a)=0$ or $g^{\prime}(b)=0$. Also, we assume that $g_{1}(x) \neq 0, x \in(a, b)$. Finally, if $\nu>0$ and $g_{1}(\xi)=0$, we assume that $f$ behaves like

$$
f(x) \sim(x-\xi)^{\nu-1+\epsilon}, \quad x \rightarrow \xi, \quad \text { with } \quad \epsilon>0 .
$$

Condition (3.5) guarantees that the integrand of $I_{H}[f]$ is integrable. Subject only to condition (3.5) and the analyticity requirements, the integration interval of (3.1) can always be split into a number of subintervals that satisfy the conditions. The assumptions guarantee that the integrand of $I_{H}[f]$ is analytic on $[a, b]$ except possibly in the points $a$ and $b$. In particular, this will allow us to apply Cauchy's integral theorem to select the integration path of (3.1).

THEOREM 3.1. Under the assumptions stated above, the integral $I_{H}[f]$ can be approximated by a sum of contributions

$$
I_{H}[f]=S^{H}[f ; a]-S^{H}[f ; b]+O\left(e^{-k P}\right),
$$

with $P>0$, and with the contributions given by the integrals

$$
S^{H}[f ; x]=\int_{0}^{P} f\left(h_{x}(p)\right) H_{\nu}^{(1)}\left(k g_{1}\left(h_{x}(p)\right)\right) e^{i k g_{2}\left(h_{x}(p)\right)} h_{x}^{\prime}(p) \mathrm{d} p,
$$

where $h_{x}(p)$ satisfies

$$
g\left(h_{x}(p)\right)=g(x)+i p
$$

The proof is almost identical to the proof of Lemma 4.1 in [18] and is omitted; it differs mainly in the special treatment of the Hankel function based on the asymptotic expression (3.2).

We note from the asymptotic behavior (3.2) that the integrand of the line integral $S^{H}[f ; x]$ in (3.7) is nonoscillatory and exponentially decaying in the integration variable $p$,

$$
H_{\nu}^{(1)}\left(k g_{1}\left(h_{x}(p)\right)\right) e^{i k g_{2}\left(h_{x}(p)\right)} \sim \sqrt{\frac{2}{\pi k g_{1}\left(h_{x}(p)\right)}} e^{i k g(x)} e^{i\left(-\frac{1}{2} \nu \pi-1 / 4 \pi\right)} e^{-k p}, \quad k \rightarrow \infty .
$$

The size of the constant $P$ is related to the size of the region of analyticity of $f$ and $g$ [18]. In the numerical examples of the scattering problem, given in section 6 , we can in fact consider the limit case $P=\infty$. The error of decomposition (3.6) then vanishes even at low frequencies.

We proceed in a way similar to that in section 2.2. Since $f$ is analytic in $D$, it has an absolutely convergent Taylor series. By the linearity of $S^{H}$, we may write

$$
S^{H}\left[f ; x_{0}\right]=\sum_{j=0}^{\infty} f^{(j)}\left(x_{0}\right) S^{H}\left[\frac{\left(x-x_{0}\right)^{j}}{j !} ; x_{0}\right] .
$$

Now, consider a subdivision of $[a, b]$ into subintervals $\left[a_{l}, b_{l}\right], l=1, \ldots, L$, such that on each subinterval the conditions of Theorem 3.1 are satisfied. Truncating the Taylor 
series of $f$ at each special point $a_{l}$ and $b_{l}$ after a finite number of terms, we arrive at a quadrature rule $Q_{H}[f]$ of the form (3.4), with weights given by

$$
\begin{aligned}
& w_{0, j}^{H}=S_{1}^{H}\left[\frac{(x-a)^{j}}{j !} ; a\right], \\
& w_{l, j}^{H}=-S_{l}^{H}\left[\frac{\left(x-x_{l}\right)^{j}}{j !} ; x_{l}\right]+S_{l+1}^{H}\left[\frac{\left(x-x_{l}\right)^{j}}{j !} ; x_{l}\right], \quad l=1, \ldots, L-1, \\
& w_{l, j}^{H}=-S_{l}^{H}\left[\frac{(x-b)^{j}}{j !} ; b\right] .
\end{aligned}
$$

The weights can be explicitly computed very efficiently, by using Gauss-Laguerre quadrature or similar techniques [11]. The accuracy of these methods improves rapidly as a function of $k$, due to the faster decay of the integrands as $k$ increases. For the purposes of our application, this advantageous characteristic is not needed. It suffices already that the number of operations for a fixed accuracy is bounded with respect to $k$. We therefore choose to focus on the convergence properties of the quadrature rule itself, rather than on the convergence of methods to compute the weights.

3.3. Convergence properties of the quadrature rule. We discuss the properties of the quadrature rule $Q_{H}[f]$, with weights given by (3.9)-(3.11), as a function of $k$. The rule is exact by construction for polynomials of degree less than or equal to

$$
p=\min _{l} d_{l} .
$$

For more general functions, the accuracy as a function of $k$ is determined by the asymptotic size of the weights. We will show that the size of the weights decreases both with increasing frequency and with increasing order of the corresponding derivative. The order of accuracy of the quadrature rule is therefore equal to the asymptotic size of the first weight that is discarded by truncation. In order to quantify this size, we require a few technical lemmas.

Lemma 3.2. Assume that $x_{0}$ is a stationary point that has order $r$. The parameterization of the path (3.8) behaves as

$$
\begin{aligned}
& h_{x_{0}}(p)=x_{0}+O\left(p^{1 /(r+1)}\right), \quad p \rightarrow 0, \\
& h_{x_{0}}^{\prime}(p)=O\left(p^{1 /(r+1)-1}\right), \quad p \rightarrow 0 .
\end{aligned}
$$

Proof. Since $g^{(j)}\left(x_{0}\right)=0, j=1, \ldots, r$, we can write the Taylor series of $g$ as

$$
g(x)=g\left(x_{0}\right)+g^{(r+1)}\left(x_{0}\right) \frac{\left(x-x_{0}\right)^{r+1}}{(r+1) !}+O\left(\left(x-x_{0}\right)^{r+2}\right) .
$$

The path $h_{x_{0}}(p)=g^{-1}\left(g\left(x_{0}\right)+i p\right)$ solves $g\left(h_{x_{0}}(p)\right)=g\left(x_{0}\right)+i p$, and hence

$$
h_{x_{0}}(p) \sim x_{0}+\sqrt[r+1]{\frac{i p(r+1) !}{g^{(r+1)}\left(x_{0}\right)}}, \quad p \rightarrow 0 .
$$

The second result follows by differentiation. Note that the complex root is multivalued: the correct root is selected by using the analytic continuation of the inverse $g_{i}^{-1}$ that satisfies $g_{i}^{-1}(g(x))=x$ on $\left[a_{i}, b_{i}\right]$ in expression (3.8).

The size of the weights follows from the size of the line integrals $S^{H}\left[\frac{\left(x-x_{0}\right)^{j}}{j !} ; x_{0}\right]$. Recall that the integral may be singular if $g_{1}(x)=0$. We will assume for the sake 
of brevity that, in that case, $g_{1}^{\prime}(x) \neq 0$. This condition is always satisfied by the applications in section 6 .

Lemma 3.3. Let $S^{H},[f ; x]$ be defined by (3.7) with $P=\infty$, and $g$ defined by (3.3). Assume that $g^{\prime}\left(x_{0}\right) \neq 0$; i.e., $x_{0}$ is not a stationary point. If $g_{1}\left(x_{0}\right) \neq 0$, we have

$$
\left|S^{H}\left[\left(x-x_{0}\right)^{j} ; x_{0}\right]\right|=O\left(k^{-j-3 / 2}\right), \quad k \rightarrow \infty .
$$

If $g_{1}\left(x_{0}\right)=0$ and $g_{1}^{\prime}\left(x_{0}\right) \neq 0$, the integral is singular and we have

$$
\left|S^{H}\left[\left(x-x_{0}\right)^{j} ; x_{0}\right]\right|=O\left(k^{-j-1}\right), \quad j \geq \nu, \quad k \rightarrow \infty .
$$

Proof. We write the integral $S^{H}\left[\left(x-x_{0}\right)^{j} ; x_{0}\right]$ as

$$
S^{H}\left[\left(x-x_{0}\right)^{j} ; x_{0}\right]=\int_{0}^{\infty} u(p) e^{-k p} \mathrm{~d} p=\frac{1}{k} \int_{0}^{\infty} u(q / k) e^{-q} \mathrm{~d} q,
$$

with

$$
u(p)=\left(h_{x_{0}}(p)-x_{0}\right)^{j} h_{x_{0}}^{\prime}(p) H_{\nu}^{(1)}\left(k g_{1}\left(h_{x_{0}}(p)\right)\right) e^{i k g_{2}\left(h_{x_{0}}(p)\right)} e^{k p} .
$$

It is a consequence of Watson's lemma that the asymptotic expansion of the integral can be obtained by integrating the asymptotic expansion of $\frac{1}{k} u(q / k)$ as $k \rightarrow \infty$, term by term in (3.16) [5,32]. Generalizing Watson's lemma, this remains true for integrals of the form $\int_{0}^{\infty} u(p) h(k p) d p$, where $h(z) \sim \log (z)^{n} z^{s} e^{-z}, n \geq 0, s \in \mathbb{Z}, z \rightarrow 0$, if the integrand is integrable [6]. This means that the singularity of the Hankel function has no influence on the asymptotic expansion.

First, consider the case $g_{1}\left(x_{0}\right) \neq 0$. Then, combining the asymptotic behavior of the Hankel function for large arguments, (3.2), with the results (3.13)-(3.14) of Lemma 3.2 for $r=0$, we have $u(q / k) \sim k^{-j-1 / 2}$. From (3.16) we can conclude $\left|S^{H}\left[\left(x-x_{0}\right)^{j} ; x_{0}\right]\right|=O\left(k^{-j-3 / 2}\right)$.

Next, consider the case $g_{1}\left(x_{0}\right)=0$. If $g_{1}^{\prime}\left(x_{0}\right) \neq 0$, then we have $g_{1}\left(h_{x_{0}}(p)\right) \sim$ $p^{1 /(r+1)}=p$. It follows that $H_{\nu}^{(1)}\left(k g_{1}\left(h_{x_{0}}(q / k)\right)\right)=O(1), k \rightarrow \infty$. Hence, by the generalization of Watson's lemma, we may conclude $\left|S^{H}\left[\left(x-x_{0}\right)^{j} ; x_{0}\right]\right|=O\left(k^{-j-1}\right)$, $j \geq \nu$.

The corresponding lemma for stationary points is very similar. The difference is due to the different behavior of the parameterization as described by Lemma 3.2.

Lemma 3.4. Let $S^{H}[f ; x]$ be defined by (3.7) with $P=\infty$, and $g$ defined by (3.3). Assume that $x_{0}$ is a stationary point of order $r$. If $g_{1}\left(x_{0}\right) \neq 0$, then we have

$$
x\left|S^{H}\left[\left(x-x_{0}\right)^{j} ; x_{0}\right]\right|=O\left(k^{-(j+1) /(r+1)-1 / 2}\right), \quad k \rightarrow \infty .
$$

If $g_{1}\left(x_{0}\right)=0$ and $g_{1}^{\prime}\left(x_{0}\right) \neq 0$, then we have

$$
\left|S^{H}\left[\left(x-x_{0}\right)^{j} ; x_{0}\right]\right|=O\left(k^{-(j+1+r / 2) /(r+1)}\right), \quad j \geq \nu, \quad k \rightarrow \infty .
$$

Proof. Consider again the function $u(p)$, given by (3.17). Assume first that $g_{1}\left(x_{0}\right) \neq 0$. We have $\left(h_{x_{0}}(q / k)-x_{0}\right)^{j} \sim k^{-j /(r+1)}$ and $h_{x_{0}}^{\prime}(q / k) \sim k^{r /(r+1)}$. Since $k g_{1}\left(h_{x_{0}}(q / k)\right) \sim k g_{1}\left(h_{x_{0}}(0)\right)=k g_{1}\left(x_{0}\right)$, we also have $H_{\nu}^{(1)}\left(k g_{1}\left(h_{x_{0}}(q / k)\right)\right) \sim k^{-1 / 2}$. Combined in (3.16), and by applying Watson's lemma, this yields the first result.

The case where $g_{1}\left(x_{0}\right)=0$ is slightly different. Since $g_{1}^{\prime}\left(x_{0}\right) \neq 0$, we have $g_{1}\left(h_{x_{0}}(q / k)\right) \sim k^{-1 /(r+1)}$ and hence $k g_{1}\left(h_{x_{0}}(q / k)\right) \sim k^{r /(r+1)}$. The Hankel function therefore yields the factor $k^{-(r / 2) /(r+1)}$ instead of $k^{-1 / 2}$ as in the first case. 
TABLE 3.1

Absolute error of the approximation of $I_{H}[f]$ by $Q_{H}[f]$, with $f(x)=\cos (x-1), g_{1}(x)=x$, and $g_{2}(x)=x^{2}+x^{3}-x$. The last row shows the value of $\log _{2}\left(e_{400} / e_{800}\right)$ : this value should approximate $d_{0} / 2+5 / 4$ (shown in parentheses).

\begin{tabular}{ccccc}
\hline$k \backslash d_{0}$ & 0 & 1 & 2 & 3 \\
\hline 100 & $1.2 E-3$ & $2.8 E-5$ & $1.3 E-6$ & $2.6 E-8$ \\
200 & $5.1 E-4$ & $8.6 E-6$ & $2.9 E-7$ & $4.1 E-9$ \\
400 & $2.2 E-4$ & $2.6 E-6$ & $6.4 E-8$ & $6.2 E-10$ \\
800 & $9.3 E-5$ & $7.8 .1 E-7$ & $1.4 E-8$ & $9.7 E-11$ \\
\hline rate & $1.23(1.25)$ & $1.73(1.75)$ & $2.20(2.25)$ & $2.68(2.75)$ \\
\hline
\end{tabular}

The convergence of the quadrature rule (3.4) as a function of $k$ can now be established. Note that the results of Lemma 3.3 agree with those of Lemma 3.4 if we take the order of a regular point to be $r=0$. Hence, we need not distinguish between stationary points and regular (end)points.

Lemma 3.5. The error of the approximation of $S_{l}^{H}\left[f ; x_{0}\right], x_{0} \in\left[a_{l}, b_{l}\right]$, is

$$
\left|S_{l}^{H}\left[f ; x_{0}\right]-Q_{l}^{S}\left[f ; x_{0}\right]\right|:=\left|S_{l}^{H}\left[f ; x_{0}\right]-\sum_{j=0}^{d_{l}} w_{l, j}^{H} f^{(j)}\left(x_{0}\right)\right|=O\left(k^{-\alpha_{l}}\right), \quad k \rightarrow \infty .
$$

If $g_{1}\left(x_{0}\right) \neq 0$, then $\alpha_{l}:=\left(d_{l}+2\right) /(r+1)-1 / 2$. If $g_{1}\left(x_{0}\right)=0$ and $g_{1}^{\prime}\left(x_{0}\right) \neq 0$, then $\alpha_{l}:=\left(d_{l}+2+r / 2\right) /(r+1)$.

Proof. Since the weights decay as a function of $k$, and as a function of the order of derivative $j$, the error of the quadrature scheme is asymptotically determined by the size of the first discarded weight. The result follows from Lemmas 3.3 and 3.4 by setting $j=d_{l}+1$.

The theorem that characterizes the accuracy of the complete quadrature rule follows immediately.

TheOREM 3.6. Consider the approximation of $I_{H}[f]$ by $Q_{H}[f]$. The error has asymptotic order $\alpha=\min _{l} \alpha_{l}$, where $\alpha_{l}$ is specified in Lemma 3.5.

As an example, we consider the integral $\int_{0}^{1} \cos (x-1) H_{0}^{(1)}(k x) e^{i k\left(x^{2}+x^{3}-x\right)} \mathrm{d} x$. The total oscillator for this integral is $g(x)=x^{2}+x^{3}$. There are two quadrature points: there is a singularity and a stationary point of order 1 at $x=0$, and a regular endpoint at $x=1$. The weights $w_{0, j}^{H}$ and $w_{1, j}^{H}$ are given by (3.9) and (3.11), respectively. From Lemma 3.4 we have $\left|w_{0, j}^{H}\right|=O\left(k^{-(j+1) / 2-1 / 4}\right)$, and from Lemma 3.3 we have $\left|w_{1, j}^{H}\right|=O\left(k^{-j-3 / 2}\right)$. Using $d_{0}$ and $d_{1}$ derivatives, the error has order $\min \left\{O\left(k^{-\left(d_{0}+2\right) / 2-1 / 4}\right), O\left(k^{-\left(d_{1}+1\right)-3 / 2}\right)\right\}$ by Theorem 3.6. We choose $d_{1}=\max \left\{0,\left\lceil\left(2 d_{0}-5\right) / 4\right\rceil\right\}$ to match the errors. Table 3.1 shows the convergence of the quadrature rule $Q_{H}[f]$ as a function of $k$ and $d_{0}$.

\section{High-frequency scattering problems.}

4.1. Problem statement. We are interested in the solution of the two-dimensional Helmholtz equation

$$
\Delta u+k^{2} u=0
$$

in the domain $\Omega^{+}=\mathbb{R}^{2} \backslash \bar{\Omega}$ that is exterior to the open domain $\Omega$, subject to a Dirichlet boundary condition $u=0$ on the boundary $\Gamma=\partial \Omega$. We assume that a periodic parameterization for $\Gamma$ is given by

$$
\kappa(\tau):[0,1] \rightarrow \Gamma \subset \mathbb{R}^{2},
$$


with $|\nabla \kappa(\tau)|>0$. The Helmholtz equation models the scattering of time-harmonic waves of the form $u(x) e^{-i \omega t}$. In the absence of damping we have $k=\omega / c$, with $c$ the speed of propagation. For the scattering of an incoming acoustic or electromagnetic wave $u^{i}(x)$ by the obstacle $\Omega$, the solution can be written as $u=u^{i}+u^{s}$. The function $u^{s}(x)$ represents the scattered wave; it satisfies the Helmholtz equation with the Dirichlet boundary condition $u^{s}(x)=-u^{i}(x)$ on $\Gamma$.

The scattered wave can be represented in terms of the single-layer potential,

$$
(S q)(x)=\int_{\Gamma} K(x, y) q(y) \mathrm{d} s_{y}, \quad \text { with } \quad K(x, y)=\frac{i}{4} H_{0}^{(1)}(k|x-y|),
$$

where $q(x)$ is the density function defined on the boundary $\Gamma$, and where $K(x, y)$ is the Green's function of the two-dimensional Helmholtz equation. The scattered wave due to an incoming wave $u^{i}$ is given by $u^{s}=-S q$ if the density function $q(x)$ is found as the solution to the combined potential integral equation

$$
\frac{q(x)}{2}+\int_{\Gamma}\left(\frac{\partial K}{\partial n_{x}}(x, y)+i \eta K(x, y)\right) q(y) \mathrm{d} s_{y}=\frac{\partial u^{i}}{\partial n}(x)+i \eta u^{i}(x), \quad x \in \Gamma,
$$

where $n_{x}$ is used to denote the normal with respect to the variable $x$. Equation (4.4) is uniquely solvable for all values of the wavenumber $k$ [8].

An important observation is that $q(x)=\frac{\partial u}{\partial n}(x)$; i.e., the density function is exactly the (exterior) normal derivative of the solution to the Helmholtz problem. This means that the solution to (4.4) is directly related to a physical property of the problem. For example, in electromagnetics, the normal derivative of the electric field is proportional to the induced current on the surface of the conducting obstacle [28].

4.2. High-frequency integral equation formulation. The density function $q(x)$ is highly oscillatory for large values of the wavenumber $k$. The solution of (4.4) therefore generally requires a large number of unknowns. In some cases, however, one has a priori information about the phase of the solution. For example, if the obstacle is convex, and if the incoming wave is a plane wave, then the phase of the solution $q$ is approximately the same as the phase of the incoming wave. Assume that the incoming wave is given by $u^{i}(x)=u_{s}^{i}(x) e^{i k g^{i}(x)}$. Then we can write

$$
q(\kappa(t))=q_{s}(t) e^{i k g^{i}(\kappa(t))}, \quad t \in[0,1],
$$

where $q_{s}(t)$ is a function that is less oscillatory than $q(\kappa(t))$, in a sense that will be made more precise in section 4.3. In physical terms, the oscillations of the induced current on a perfectly conducting surface tend to follow the oscillations of the incoming electromagnetic wave. This is the main reason why the problem is formulated such that the solution $q(x)$ corresponds to a physical variable - only in that case is the phase known in the form of (4.5). This was noted in [7]; the integral equation formulation of this section follows the same pattern as in [7].

Substituting the ansatz (4.5) into (4.4) leads to the integral equation of the second kind

$$
\frac{1}{2} q_{s}(t) e^{i k g^{i}(\kappa(t))}+\int_{0}^{1} G(t, \tau) q_{s}(\kappa(\tau)) e^{i k g^{i}(\kappa(\tau))} \mathrm{d} \tau=v^{i}(\kappa(t)),
$$

with

$$
G(t, \tau):=\left(\frac{\partial K}{\partial n_{x}}(\kappa(t), \kappa(\tau))+i \eta K(\kappa(t), \kappa(\tau))\right)|\nabla \kappa(\tau)|
$$




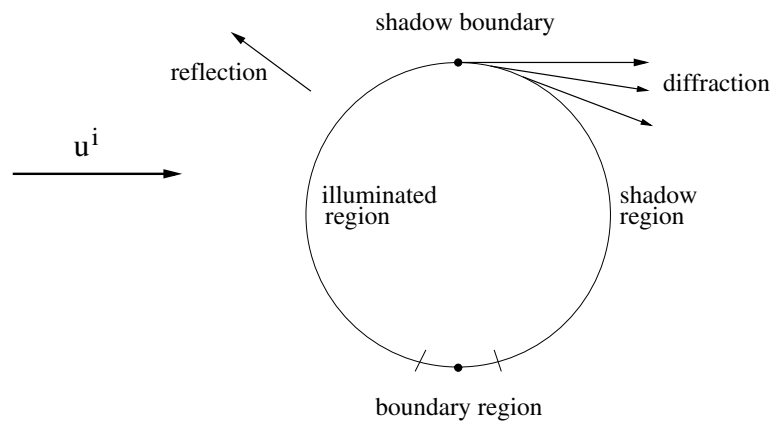

FIG. 4.1. Reflection and diffraction effects in the scattering of an incoming wave $u^{i}$ by a smooth and convex obstacle.

and with the right-hand side given by

$$
v^{i}(x):=\frac{\partial u^{i}}{\partial n}(x)+i \eta u^{i}(x) .
$$

The unknown in (4.6) is the function $q_{s}(t)$. Since $q_{s}(t)$ is less oscillatory than the original density function $q(\kappa(t))$, one can solve (4.6) using a coarser discretization. In the next section, we discuss the remaining oscillatory behavior of $q_{s}(t)$.

4.3. Asymptotic behavior of the solution. In the past decades, a lot of effort has been invested in studying the asymptotic behavior of the solution $q(x)$ to (4.4) as a function of the wavenumber, concentrating mainly on the scattering of a plane wave (see, e.g.,[23, 27] and references therein). For smooth and convex obstacles, there are three important regions with different properties, illustrated in Figure 4.1: the illuminated region, the shadow region, and the transitional shadow boundary region. In the illuminated region, the scattered wave is described asymptotically by geometrical optics: a wave is reflected such that the angle of incidence and the angle of reflection are identical. A wave tangential to the shadow boundary is diffracted. The density function decays rapidly away from the shadow boundary into the shadow region, due to the continuous emission of diffracted waves. In the shadow region the function $q(x)$ approaches zero as $k$ increases.

The asymptotic expansion of $q_{s}(t)$ for large $k$ reflects these three regions. Assume an incoming plane wave in the direction $\alpha$ of the form $u^{i}(x)=e^{i k \alpha \cdot x}$, with $|\alpha|=1$. It was proved in [27] that $q_{s}(t)$ has a uniform asymptotic expansion of the form

$$
q_{s}(t) \sim \sum_{m, n \geq 0} k^{2 / 3-n-2 m / 3} b_{m, n}(\alpha, t) \Psi^{(n)}\left(k^{1 / 3} Z(\alpha, t)\right),
$$

which remains valid as $t$ crosses the shadow boundaries. The shadow boundary points can be characterized here by $\alpha \cdot \nu=0$, with $\nu$ the exterior normal to $\Omega$. For a thorough discussion and analysis of this expansion, we refer the reader to [12]. Here, we only recall the main characteristics of the expansion, as a means to motivate the choices that are made later on.

The function $Z \in C^{\infty}$ is infinitely smooth and has a simple root at the two shadow boundary points. The function $Z$ is positive when $\alpha \cdot \nu<0$, i.e., in the illuminated region, and it is negative in the shadow region. The function $\Psi(z)$ is smooth for positive arguments, with

$$
\Psi(z) \sim z, \quad z \rightarrow \infty
$$


and it is decaying rapidly but in an oscillatory manner for large negative arguments. It follows that the function $q_{s}(t)$ is smooth in the illuminated region but may be oscillatory in the shadow region, corresponding to the behavior of $\Psi(z)$ for large positive arguments and large negative arguments, respectively. One can also deduce the following asymptotic properties from the leading order of (4.7):

$$
\left|q_{s}(t)\right|=\left\{\begin{array}{cc}
O(k), & \text { illuminated region, } \\
O\left(k^{2 / 3}\right), & \text { shadow boundary. }
\end{array}\right.
$$

There is a $k$-dependent transition region near the shadow boundary. Motivated by (4.7), we introduce a transition region of size $O\left(k^{-1 / 3}\right)$ around the shadow boundary points, and define the shadow boundary regions as

$$
\begin{aligned}
& T_{B 1}(k)=\left[t_{s b 1}-D_{1} k^{-1 / 3}, t_{s b 1}+C_{1} k^{-1 / 3}\right], \\
& T_{B 2}(k)=\left[t_{s b 2}-C_{2} k^{-1 / 3}, t_{s b 2}+D_{2} k^{-1 / 3}\right],
\end{aligned}
$$

with constants $C_{1}, C_{2}, D_{1}, D_{2}>0$ independent of $k$, but small enough such that $T_{B 1}(k)$ and $T_{B 2}(k)$ are nonoverlapping, and with $t_{s b 1}$ and $t_{s b 2}$ the locations of the two shadow boundary points in the parameter domain $[0,1]$. The illuminated region is defined as

$$
T_{I}(k)=\left(t_{s b 1}+C_{1} k^{-1 / 3}, t_{s b 2}-C_{2} k^{-1 / 3}\right) .
$$

The shadow region is the remaining part of the interval $[0,1]$.

The size of the transition region is related to the behavior of the argument $k^{1 / 3} Z(\alpha, t)$ of the function $\Psi(z)$ in (4.7). Because $Z(\alpha, t)$ has a simple zero at $t_{s b 1}$, we have

$$
Z(\alpha, t) \approx Z^{\prime}\left(\alpha, t_{s b 1}\right)\left(t-t_{s b 1}\right), \quad t \rightarrow t_{s b 1} .
$$

Hence, there exists a constant $c$ such that for sufficiently large $k$ we have

$$
\left|k^{1 / 3} Z(\alpha, t)\right| \leq c \quad \text { if } t \in T_{B 1}(k) .
$$

A similar bound holds for the region $T_{B 2}(k)$. Since the oscillations of $q_{s}(t)$ originate in the oscillatory behavior of $\Psi(z)$ for negative arguments, the meaning of the bound (4.13) is that the number of oscillations of $q_{s}(t)$ in the shadow boundary region $T_{B 1}(k)$ is bounded in $k$.

Remark 4.1. The remaining oscillatory behavior of $q_{c}(t)$ in the shadow boundary region indicates that the oscillations of the incoming wave do not completely describe the oscillatory behavior of $q(x)$. Still, we will see that the ansatz (4.5) is suitable for further computations.

5. A hybrid high-frequency boundary element method. The collocation of integral equation (4.6) in a point $x_{n}$ leads to a one-dimensional and oscillatory integral in the integration variable $\tau$. In this section, we show how an efficient quadrature rule can be used for the discretization of that collocation integral. First, we discuss the classical boundary element approach in section 5.1. The application of specialized quadrature rules, leading to a highly sparse discretization matrix, is described in section 5.2. We comment on the location of the quadrature points section 5.3 and on the limitations of the approach in section 5.4. It turns out that the quadrature rule can not be applied everywhere. Finally, we arrive at a method combining a sparse discretization with a dense part in section 5.5. 
5.1. Collocation approach for the discretization. Consider a collocation scheme for integral equation (4.6), with a set of $N$ distinct collocation points $x_{n}=$ $\kappa\left(t_{n}\right), t_{n} \in[0,1], n=1, \ldots, N$. The classical way to proceed is to look for an approximation $q_{c}$ to solution $q_{s}$ in the form

$$
q_{c}(t)=\sum_{m=1}^{N} c_{m} \phi_{m}(t),
$$

where the $\phi_{m}$ functions are a set of linearly independent basis functions with support $\Omega_{m}:=\operatorname{supp}\left(\phi_{m}\right)$. The number of basis functions may be small, since the exact solution $q_{s}$ is not very oscillatory. Collocating (4.6) in the points $t_{n}$, with $q_{s}$ replaced by $q_{c}$, leads to the equations

$$
\frac{1}{2} q_{c}\left(t_{n}\right) e^{i k g^{i}\left(x_{n}\right)}+\int_{0}^{1} G\left(t_{n}, \tau\right) q_{c}(\tau) e^{i k g^{i}(\kappa(\tau))} \mathrm{d} \tau=v^{i}\left(x_{n}\right), \quad n=1, \ldots, N .
$$

The collocation approach therefore leads to a linear system $A c=b$ of size $N \times N$, where the elements of the discretization matrix $A$ are given by

$$
A_{n, m}=\frac{1}{2} \phi_{m}\left(t_{n}\right) e^{i k g^{i}\left(\kappa\left(t_{n}\right)\right)}+\int_{\Omega_{m}} G\left(t_{n}, \tau\right) e^{i k g^{i}(\kappa(\tau))} \phi_{m}(\tau) \mathrm{d} \tau
$$

and the right-hand side by $b_{n}=v^{i}\left(x_{n}\right)$. The discretization matrix $A$ is dense, but small compared to the classical boundary element discretization for the original equation. Hence, this is a big improvement over the direct discretization of (4.4). Since the elements (5.3) are given by oscillatory integrals, they can be computed efficiently using the numerical steepest descent technique described in section 2.1. This would yield an efficient total solution method, which remains efficient when $k$ increases, but that is also accurate for small values of $k$.

However, there are still some issues associated with this approach. Since the matrix is dense, the method requires the evaluation of $N^{2}$ integrals. Although $N$ may be rather small, the computational cost can still be high. Interestingly, it was observed in [16] that many of the elements are small and can in fact be discarded, reducing the computation time. A second, more important issue is that the results of [16] indicate that the error of the scheme increases with increasing wavenumber. Here, we examine a different discretization of (5.2) that aims to address these issues, based on the quadrature rule developed in section 3 and motivated by the accuracy of this rule for high wavenumbers. Owing to the small number of required quadrature points, the resulting discretization matrix will be highly sparse.

5.2. The application of specialized quadrature rules. Based on the collocation integral (5.2), we define the following general oscillatory integral:

$$
I_{c}\left[f ; t_{n}\right]:=\int_{0}^{1} f(\tau) G\left(t_{n}, \tau\right) e^{i k g^{i}(\kappa(\tau))} \mathrm{d} \tau .
$$

The oscillatory function $G(t, \tau)$ in the integrand is given explicitly by

$$
\begin{aligned}
G(t, \tau)= & -\frac{i}{4} k \frac{\nabla \kappa(\tau) \cdot(\kappa(t)-\kappa(\tau))}{|\kappa(t)-\kappa(\tau)|}|\nabla \kappa(\tau)| H_{1}^{(1)}(k|\kappa(t)-\kappa(\tau)|) \\
& -\frac{\eta}{4}|\nabla \kappa(\tau)| H_{0}^{(1)}(k|\kappa(t)-\kappa(\tau)|) .
\end{aligned}
$$


Choosing $\eta=k$, this function can further be written as

$$
G(t, \tau)=a(\tau) k H_{0}^{(1)}(k|\kappa(t)-\kappa(\tau)|)+b(t, \tau) k H_{1}^{(1)}(k|\kappa(t)-\kappa(\tau)|),
$$

with functions $a(\tau)$ and $b(t, \tau)$ that are independent of $k$. Hence, integral (5.4) can be written as a sum of two model integrals of the form $I_{H}[f]$, involving Hankel functions, multiplied by $k$. Both model integrals have the same oscillator,

$$
g\left(\tau ; t_{n}\right):=\left|\kappa\left(t_{n}\right)-\kappa(\tau)\right|+g^{i}(\kappa(\tau)) .
$$

In particular, this means that one can find a quadrature rule such that

$$
I_{c}\left[f ; t_{n}\right] \approx Q_{c}\left[f ; t_{n}\right]:=\sum_{l=0}^{L_{n}} \sum_{j=0}^{d_{n, l}} w_{n, l, j}^{c} f^{(j)}\left(\tau_{n, l}\right) .
$$

The rule $Q_{c}\left[f ; t_{n}\right]$ is computed as follows:

- The weights $w_{n, l, j}^{c}$ are given by the expressions (3.9)-(3.11) for the weights $w_{l, j}^{H}$ of the model integral, with the line integrals $S^{H}[f ; x]$ replaced by

$$
S^{c}[f ; x]:=\int_{0}^{P} f\left(h_{x}(p)\right) G\left(t_{n}, h_{x}(p)\right) e^{i k g^{i}\left(\kappa\left(h_{x}(p)\right)\right.} h_{x}^{\prime}(p) \mathrm{d} p,
$$

where the integration path is the path of steepest descent corresponding to the oscillator (5.6). Compared to $S^{H}[f ; x]$, we have included the additional factors $k a(\tau)$ and $k b(t, \tau)$ from (5.5) into the weight function of the integral. We note that it implies the assumption that $\kappa(\tau)$ is analytic. The scaling by a factor $k$ reduces the asymptotic order, based on the absolute error, by 1 .

- The quadrature points $\tau_{n, l}$ are found from the oscillator (5.6). They are the points where the integrand becomes singular (and hence nonanalytic) and the stationary points of the oscillator $g\left(\tau ; t_{n}\right)$. These points are derived by a straightforward but technical analysis of $g\left(\tau ; t_{n}\right)$. There are no contributing endpoints, as the integrand is periodic on the closed curve $\Gamma$.

We now describe how the quadrature rule (5.7) can be used in the discretization. The derivatives of $q_{c}$ can be written in terms of the basis functions $\phi_{m}$,

$$
q_{c}^{(j)}(\tau)=\sum_{m=1}^{N} c_{m} \phi_{m}^{(j)}(\tau)
$$

Hence, applying the quadrature rule to $q_{c}$ yields a matrix $B$ with entries

$$
B_{n, m}=\left\{\begin{array}{cc}
\sum_{l: \tau_{n, l} \in \Omega_{m}} \sum_{j=0}^{d_{n, l}} w_{n, l, j}^{c} \phi_{m}^{(j)}\left(\tau_{n, l}\right), & \exists l \in\left[0, L_{n}\right]: \tau_{n, l} \in \Omega_{m}, \\
0 & \text { otherwise. }
\end{array}\right.
$$

The entry $B_{n, m}$ is nonzero only if at least one quadrature point $\tau_{n, l}$ exists that lies in the support of the basis function $\phi_{m}$. The number of nonzero points therefore depends on the size of the supports of the basis functions. If all basis functions are local, then the matrix $B$ is highly sparse.

Remark 5.1. The asymptotic order of quadrature rule (5.7) is limited by the number of derivatives $d_{n, l}$ that are used, as described by Theorem 3.6. In turn, note from (5.9) that $d_{n, l}$ is limited by the number of derivatives that exist for the chosen basis functions. 


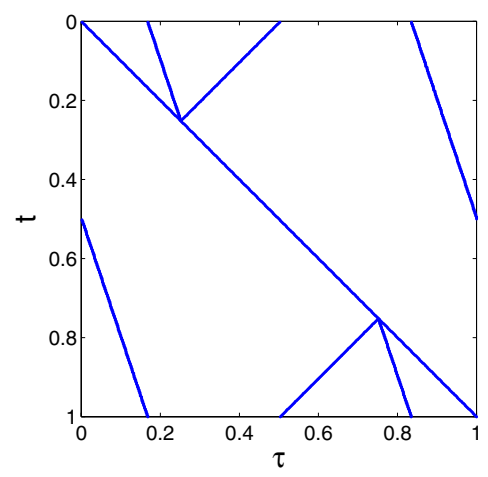

FIG. 5.1. The location of the contributing points of the collocation integral for the scattering of a plane wave by a circular obstacle. Each row corresponds to a fixed value $t \in[0,1]$, with the shadow boundary points at 0.25 and 0.75 , and the illuminated region in between. The singular points are located along the diagonal. The off-diagonal points correspond to stationary points.

5.3. Location of the quadrature points. The location of the quadrature points is illustrated in Figure 5.1 for the scattering of a plane wave by a circular obstacle. There is one stationary point if $t_{n}$ lies in the illuminated region, and there are three stationary points if $t_{n}$ lies in the shadow region. Two of these points coalesce into one stationary point of order $r=2$ exactly at the shadow boundary. Using local basis functions, the structure of the discretization matrix $B$ closely resembles that of Figure 5.1.

The coalescence of two stationary points at the shadow boundary is a general property for convex obstacles [22]. The quadrature points on the diagonal reflect the general principle of localization of high-frequency scattering, which states that the scattering of a ray is asymptotically determined by properties local to the point of incidence [13]. As such, Figure 5.1 is illustrative for more general convex shapes.

Remark 5.2. Figure 5.1 shows the existence of a stationary point in the shadow region when $t \in T_{I}(k)$ is a point in the illuminated region. This seems to contradict the localization principle. Indeed, this stationary point has no physical relevance for the scattering problem. Following Remark 4.1, it is an artifact of the incorrect ansatz in the shadow region.

5.4. Limitations of the sparse discretization. The convergence of quadrature rule $Q_{c}\left[f ; t_{n}\right]$ as a function of $k$ is described by the corresponding results for $Q_{H}[f]$ discussed in section 3.3. In particular, the results imply that the accuracy of the approximation increases with increasing $k$, at a rate that depends on the number of derivatives that are used in the quadrature rule. For example, for $t \in T_{I}(k)$, we have

$$
\left|I_{c}\left[f ; t_{n}\right]-Q_{c}\left[f ; t_{n}\right]\right|=O\left(k^{-\min \left\{d_{n, 0}+1,\left(d_{n, 1}+2\right) / 2-3 / 2\right\}}\right),
$$

where $\tau_{n, 0}=t_{n}$ and $\tau_{n, 1}$ is the stationary point of order $r=1$ in the shadow region. Hence, the sparse discretization matrix $B$ is very well suited for computing the application of the integral operator under consideration to a function of the form $f(t) e^{i k g^{i}(\kappa(t))}$.

The convergence results in section 3.3 were derived, however, with the assumption that the function $f$ is independent of $k$. No statements can therefore be made 


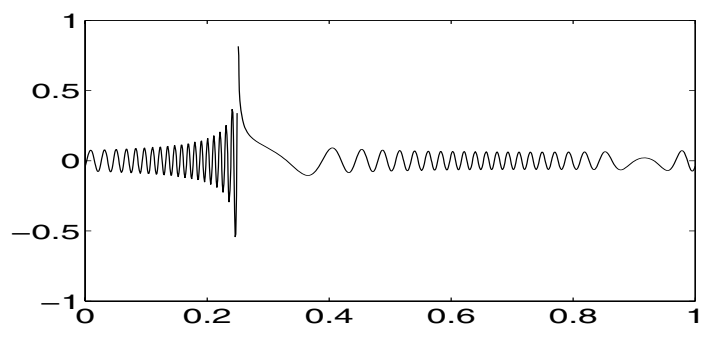

FIG. 5.2. The real part of the integrand for $t_{n}=t_{s b 1}$ for a circular obstacle and $k=100$.

regarding the computed solution of an integral equation using the sparse discretization matrix $B$, because the solution generally depends on $k$. Moreover, it was shown that the assumed oscillatory behavior $e^{i k g^{i}(\kappa(t))}$ in the ansatz (4.5) does not fully describe the oscillatory behavior of the exact solution $q(x)$. Therefore, $Q_{c}\left[q_{s} ; t_{n}\right]$ might not be a good approximation to $I_{c}\left[q_{s} ; t_{n}\right]$. It is important that the quadrature rule be accurate when applied to the exact solution $q_{s}$. Otherwise, one cannot expect that solving the system with the sparse representation matrix $B$ can yield a good approximation of the exact solution.

It can be verified that $Q_{c}\left[q_{s} ; t_{n}\right]$ is accurate when $t_{n} \in T_{I}(k)$, i.e., when $t_{n}$ lies in the illuminated region. The cases $t \in T_{s b 1}(k)$ and $t \in T_{s b 2}(k)$ are more problematic. Figure 5.2 shows the real part of the integrand of the collocation integral $I_{c}\left[1 ; t_{s b 1}\right]$, where $t_{n}=t_{s b 1}$ coincides with the first shadow boundary. This integral has a stationary point of order $r=2$ at $t_{s b 1}$, but only to the right of the singularity. The integral is highly oscillatory to the left of the shadow boundary, and not oscillatory to the right. It turns out that a quadrature rule similar to $Q_{c}\left[f ; t_{n}\right]$ can be used on the left interval $\left[0, t_{s b 1}\right]$ and in the illuminated region $T_{I}(k)$. It is not suited for the intermediate interval $\left[t_{s b 1}, t_{s b 1}+C_{1} k^{-1 / 3}\right]$. Due to the stationary point, however, the integrand is not oscillatory in that interval. In the following section, we will construct a sparse approximation matrix that is based on the use of specialized quadrature rules in the regions where they apply. They are combined with a classical dense discretization in the remaining part of the integration domain.

Remark 5.3. The failure of $Q_{c}\left[q_{s} ; t_{s b 1}\right]$ for the exact solution $q_{s}$ can be seen as follows: the asymptotic decay of the quadrature weights $w_{n, l, j}$ for increasing $k$ is cancelled exactly by the growth of the derivatives $q_{s}^{(j)}\left(t_{s b 1}\right)$ for increasing $k$. Hence, the rule does not converge.

\subsection{A sparse discretization for the scattering problem.}

Basis functions and collocation points. The basis functions for the discretization are chosen corresponding to the behavior of the solution in the three different regions identified in section 4.3. Recall that the solution is smooth in the illuminated region $T_{I}(k)$, and oscillatory but rapidly decaying in the shadow region. First, following [7], we approximate the solution by zero in the shadow region. We choose a fixed number of basis functions in the illuminated region $T_{I}(k)$. Finally, we also choose a fixed number of basis functions in the transitional shadow boundary regions $T_{B 1}(k)$ and $T_{B 2}(k)$, independently of $k$. This corresponds to using a fixed number of basis functions per oscillation of the solution in these regions.

The collocation points $V$ are chosen equidistantly in the regions $T_{B 1}(k), T_{B 2}(k)$, 


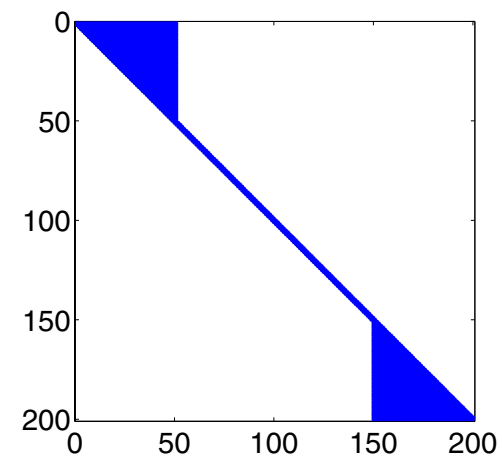

FIG. 5.3. Illustration of the sparse discretization matrix using cubic B-splines for the scattering of a plane wave by a circular obstacle. The middle part of the matrix is tridiagonal.

and $T_{I}(k)$. We have $V=V_{1} \cup V_{2} \cup V_{3}$, where

$$
\begin{aligned}
& V_{1}:=\left\{t_{s b 1}-D_{1} k^{-1 / 3}+j h_{1}\right\}_{j=0}^{N_{1}}, \quad \text { with } h_{1}:=\frac{\left(C_{1}+D_{1}\right) k^{-1 / 3}}{N_{1}}, \\
& V_{2}:=\left\{t_{s b 1}+C_{1} k^{-1 / 3}+j h_{2}\right\}_{j=1}^{N_{2}-2}, \quad \text { with } h_{2}:=\frac{\left(t_{s b 2}-t_{s b 1}-\left(C_{1}+C_{2}\right) k^{-1 / 3}\right)}{N_{2}-1}, \\
& V_{3}:=\left\{t_{s b 2}-C_{2} k^{-1 / 3}+j h_{3}\right\}_{j=0}^{N_{3}}, \quad \text { with } h_{3}:=\frac{\left(C_{2}+D_{2}\right) k^{-1 / 3}}{N_{3}} .
\end{aligned}
$$

These are $N=N_{1}+N_{2}+N_{3}$ points. As basis functions, we choose B-splines of odd degree $s$ with knots $U$ given by

$$
U:=V \cup\left\{t_{s b 1}-D_{1} k^{-1 / 3}-j h_{1}\right\}_{j=1}^{(s+1) / 2} \cup\left\{t_{s b 2}+D_{1} k^{-1 / 3}+j h_{3}\right\}_{j=1}^{(s+1) / 2} .
$$

These $N+s+1$ points uniquely determine $N$ distinct B-splines. The basis functions are $s-1$ times continuously differentiable. The nodes in the shadow region are added in order to ensure a smooth transition into the shadow region where the solution is approximated by zero.

Discretization. For the discretization, we propose the following scheme. If $t_{n} \in$ $T_{I}(k)$, a collocation point in the illuminated region, then

- the quadrature rule $Q_{c}\left[f ; t_{n}\right]$ is applied for the singular point $t_{n}$,

- the stationary points lie in the shadow region, and they are discarded.

For splines of degree $s$, the singular point $t_{n}$ lies in the support of only $s$ separate basis functions. The corresponding matrix entries are given by (5.10). The contributions of the stationary points are discarded because the solution is approximated by zero in the shadow region. Recall that in the context of the scattering problem, following Remark 5.2 , these are only spurious stationary points.

If $t_{n} \in T_{B 1}(k)$, corresponding to the first shadow boundary region, then

- a specialized quadrature rule for oscillatory integrals is applied on the interval $\left[0, t_{n}\right]$

- a classical dense discretization is used on the interval $\left[t_{n}, t_{s b 1}+C_{1} k^{-1 / 3}\right]$,

- a specialized quadrature rule for oscillatory integrals is again applied on the interval $\left[t_{s b 1}+C_{1} k^{-1 / 3}, 1\right]$. 
The quadrature rule on $\left[0, t_{n}\right]$ reduces to the contribution of the singular point $t_{n}$. The corresponding weights have the form of (3.11) with $S_{l}^{H}$ replaced by $S_{l}^{c}$,

$$
w_{n, l_{n}, j}^{c}=-S_{l_{n}}^{c}\left[\frac{\left(x-t_{n}\right)^{j}}{j !} ; t_{n}\right],
$$

where $l_{n}$ is the index such that $t_{n}=\tau_{n, l_{n}}$. The quadrature rule on $\left[t_{s b 1}+C_{1} k^{-1 / 3}, 1\right]$ consists of the contributions of the stationary points $\tau_{n, l}$ outside the shadow boundary region, and of the endpoint $t_{r}:=t_{s b 1}+C_{1} k^{-1 / 3}$. The weights corresponding to that endpoint have the form of (3.9),

$$
w_{n, l_{r}, j}^{c}=S_{l_{r}+1}^{c}\left[\frac{\left(x-t_{r}\right)^{j}}{j !} ; t_{r}\right],
$$

where $l_{r}$ is the index such that $t_{r} \in\left[\tau_{n, l_{r}}, \tau_{n, l_{r}+1}\right]$. Finally, the dense discretization in the interval $\left[t_{n}, t_{r}\right]$ leads to elements of the form

$$
\sigma_{n, m}=\frac{1}{2} \phi_{m}\left(t_{n}\right) e^{i k g^{i}\left(x_{n}\right)}+\int_{\Omega_{m} \cap\left[t_{n}, t_{r}\right]} G\left(t_{n}, \tau\right) e^{i k g^{i}(\kappa(\tau))} \phi_{m}(\tau) \mathrm{d} \tau .
$$

The only difference compared to (5.3) is that the integration domain may be cut at the boundaries of $\left[t_{n}, t_{r}\right]$. Summarizing, the elements of the discretization matrix for $t_{n} \in T_{B 1}(k)$ can be written as

$$
C_{n, m}=\left\{\begin{array}{cc}
\sigma_{n, m} & \text { if } \Omega_{m} \cap\left[t_{n}, t_{r}\right] \neq \varnothing \\
+\sum_{j=0}^{d_{n, l_{n}}} w_{n, l_{n}, j}^{c} \phi_{m}^{(j)}\left(t_{n}\right) & \text { if } t_{n} \in \Omega_{m} \\
+\sum_{j=0}^{d_{n, l_{r}}} w_{n, l_{r}, j}^{c} \phi_{m}^{(j)}\left(t_{r}\right) & \text { if } t_{r} \in \Omega_{m} \\
\quad+\sum_{l: t_{r}<\tau_{n, l} \in \Omega_{m}} \sum_{j=0}^{d_{n, l}} w_{n, l, j}^{c} \phi_{m}^{(j)}\left(\tau_{n, l}\right), & \exists l \in\left[0, L_{n}\right]: t_{r}<\tau_{n, l} \in \Omega_{m}, \\
\sum_{l: t_{r}<\tau_{n, l} \in \Omega_{m}} \sum_{j=0}^{d_{n, l}} w_{n, l, j}^{c} \phi_{m}^{(j)}\left(\tau_{n, l}\right), & \exists l \in\left[0, L_{n}\right]: t_{r}<\tau_{n, l} \in \Omega_{m}, \\
0 & \text { otherwise. }
\end{array}\right.
$$

The case $t_{n} \in T_{B 2}(k)$ can be treated similarly. The structure of the sparse matrix $C$ is illustrated in Figure 5.3. The two small dense parts correspond to the dense discretization in the intervals $\left[t_{n}, t_{s b 1}+C_{1} k^{-1 / 3}\right]$ and $\left[t_{s b 2}-C_{2} k^{-1 / 3}, t_{s b 2}\right]$. For simplicity, we have chosen the constant $C_{1}$ large enough such that, for $t_{n} \in T_{B 1}$, all stationary points $\tau_{n, l} \in T_{B 1}(k)$ also lie in the shadow boundary region. The constant $C_{2}$ was chosen similarly.

One can verify that the required integrals of the form (5.13) are not oscillatory. Due to the stationary point of order $r=2$, the integrand behaves as $e^{i k c\left(\tau-t_{s b 1}\right)^{3}}$ near the shadow boundary. The argument of the exponential is bounded in $k$, since by construction we have

$$
\left|\tau-t_{s b 1}\right| \leq \max \left\{C_{1}, D_{1}\right\} k^{-1 / 3} .
$$

Hence, there is only a bounded number of oscillations in the integrals for increasing $k$. It is therefore reasonable to expect that the integrals can be evaluated with a number of operations that is independent of $k$. Since the weights of the quadrature rule can be evaluated efficiently as well, and because the number of unknowns is fixed, the matrix in Figure 5.3 can be computed with a total number of operations that is independent of $k$. 


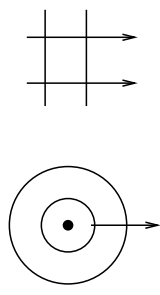

$(-2,0)$

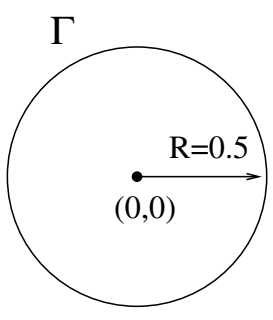

(a) Circle

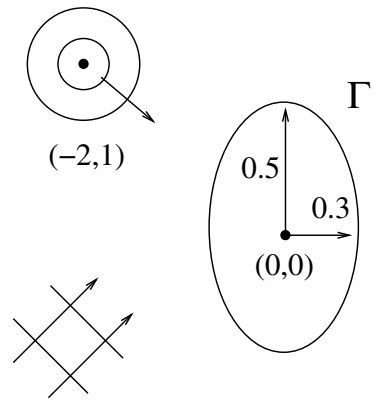

(b) Ellipse

FIG. 6.1. Illustration of two smooth convex scattering obstacles. The boundary conditions are plane waves, or circular waves originating from a point source.

6. Numerical results. We consider the scattering by two convex obstacles, a circle and an ellipse, shown in Figure 6.1. We use two types of boundary conditions: a plane wave, modeled in the form $u^{i}(x)=e^{i k \alpha \cdot x}$, and a point source, modeled by $u^{i}(x)=H_{0}^{(1)}\left(\left|x-x_{0}\right|\right)$, with $x_{0}$ a point in the exterior $\Omega^{+}$of the obstacle. The circle and ellipse are parameterized by

$$
\kappa(t)=\left\{\begin{array}{l}
R \cos (2 \pi t), \\
R \sin (2 \pi t),
\end{array} \text { and } \quad \kappa(t)=\left\{\begin{array}{l}
R_{1} \cos (2 \pi t), \\
R_{2} \sin (2 \pi t),
\end{array}\right.\right.
$$

respectively.

In all examples, we have chosen the constants $C_{1}$ and $C_{2}$ large enough (w.r.t. $D_{1}$ and $D_{2}$ ) such that the sparse matrix has the form shown in Figure 5.3. The highest order of the derivative used in the specialized quadrature rule for oscillatory integrals is kept fixed, $d_{n, l}=d, \forall n, l$, with $d \leq s-1$, where $s$ is the degree of the piecewise polynomial spline basis. The integrals (5.13) for the elements of the dense parts that are singular were evaluated using Cubpack [9].

6.1. Total solution time. The smooth function $q_{c}(t)$ is illustrated in Figure 6.2 as it was computed for the different scattering problems. The mild oscillatory behavior of the function near the shadow boundary is illustrated in the left panel of Figure 6.3, showing only the real part of the solution. Two spikes are present near the shadow boundary, with a peak value that scales as $O\left(k^{2 / 3}\right)$, as predicted by the estimate (4.9). The dashed line shows the effect of doubling $k$. The $O(k)$ behavior in the illuminated region is clear from the imaginary part illustrated in the right panel of Figure 6.3.

Table 6.1 shows the timings for an implementation of the algorithm of section 5.5 in Matlab. In all the examples considered, the time actually decreases with increasing wavenumber $k$. This is due to the fact that, at larger frequencies, the weights of the specialized quadrature rule $Q_{c}\left[f ; t_{n}\right]$ are easier to compute. Also, the integrals (5.13) that determine the elements of the dense parts do not become more oscillatory. In a classical boundary element method, and using 10 unknowns per wavelength, the case $k=10^{5}$ would correspond to a dense matrix with approximately $N=0.5 \times 10^{6}$ unknowns.

6.2. Error and convergence. In this section, we present numerical results evaluating the error of the proposed scheme. First, we investigate the absolute and 


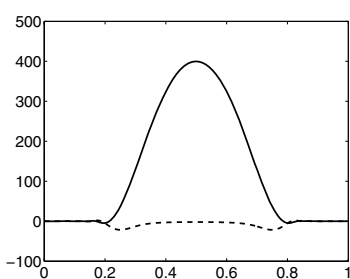

(a) Circle, plane wave

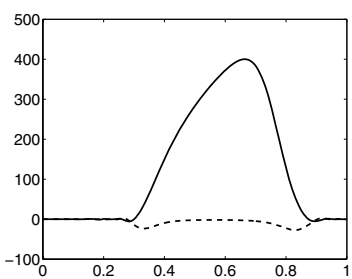

(c) Ellipse, plane wave

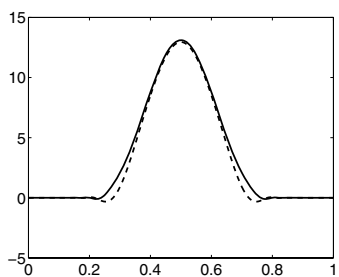

(b) Circle, point source

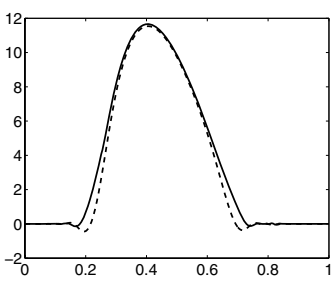

(d) Ellipse, point source

FIG. 6.2. The real part (dashed lines) and imaginary part (solid lines) of the computed smooth function $q_{c}(t)$. The parameters correspond to the case $k=200$ in Table 6.1.
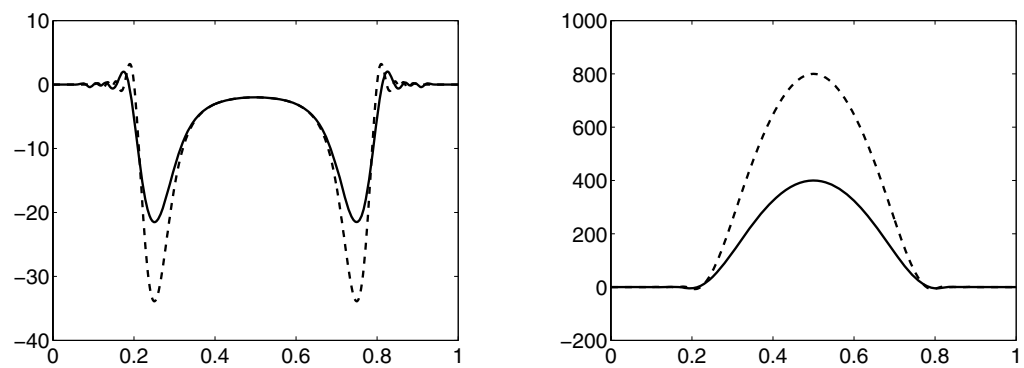

FIG. 6.3. The real part (left) and imaginary part (right) of $q_{c}(t)$ for the scattering of a plane wave by a circle. The solid lines correspond to $k=200$, the dashed lines to $k=400$.

relative error for increasing values of $k$. Next, we show that the accuracy is improved by increasing the number of derivatives $d$ that are used in the specialized quadrature rules. We then illustrate the influence of the constants $C_{1}, C_{2}, D_{1}$, and $D_{2}$ that determine the size of the shadow boundary region. Finally, we illustrate the influence of the parameters $N_{1}, N_{2}$, and $N_{3}$ that determine the total degrees of freedom.

In applications, one is usually interested in the quantity $q_{s} / k$. For example, in electromagnetics, this quantity is proportional to the induced current on the surface of the obstacle with a proportionality constant that is independent of $k$. One can see from (4.9) that $q_{s} / k$ corresponds to a normalization of $q_{s}$ for different $k$. For this 
TABLE 6.1

Total solution time in seconds for the different scattering problems. All parameters are kept fixed, except the wavenumber $k$. We used $d=2$ derivatives in the quadrature rules.

\begin{tabular}{lcccc}
\hline & \multicolumn{2}{c}{ Circle } & \multicolumn{2}{c}{ Ellipse } \\
& Plane wave & Point source & Plane wave & Point source \\
\hline$\left(C_{1}, C_{2}\right)$ & $(1.3,1.3)$ & $(1,0.1 .0)$ & $(1.5,1.3)$ & $(1.3,1.3)$ \\
$\left(D_{1}, D_{2}\right)$ & $(1.0,1.0)$ & $(0.8,0.8)$ & $(1.3,1.0)$ & $(1.0,1.0)$ \\
$\left(N_{1}, N_{2}, N_{3}\right)$ & $(30,30,30)$ & $(30,30,30)$ & $(60,30,60)$ & $(60,30,60)$ \\
\hline$k=200$ & $287 s$ & $312 s$ & $463 s$ & $512 s$ \\
$k=400$ & $283 s$ & $308 s$ & $450 s$ & $496 s$ \\
$k=800$ & $281 s$ & $306 s$ & $443 s$ & $491 s$ \\
$k=1600$ & $279 s$ & $302 s$ & $438 s$ & $484 s$ \\
$k=10000$ & $273 s$ & $294 s$ & $422 s$ & $470 s$ \\
$k=100000$ & $269 s$ & $289 s$ & $416 s$ & $459 s$ \\
\hline
\end{tabular}

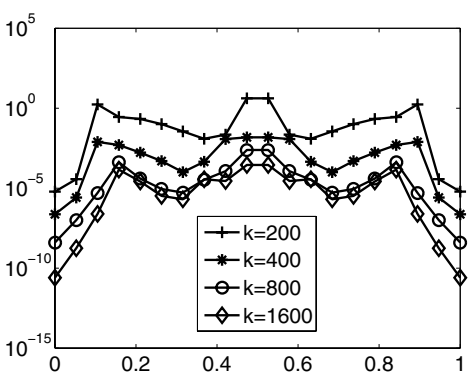

(a) Absolute error $E_{a}$

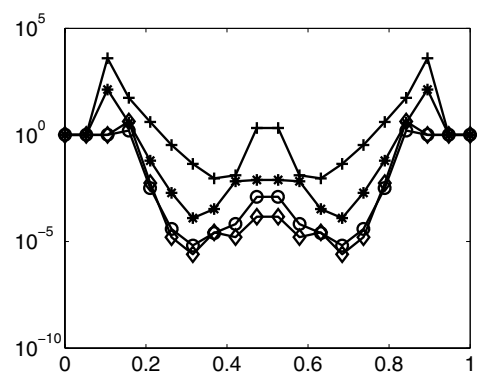

(b) Relative error $E_{r}$

FIG. 6.4. Absolute and relative error for the scattering of a plane wave by a circle for different values of $k$. We have used $d=1$ derivative in the specialized quadrature rules. The other parameters correspond to those in Table 6.1.

reason, in all following results we evaluate the absolute error in a point $t$ as

$$
E_{a}(t)=\frac{\left|q_{s}(t)-q_{c}(t)\right|}{k},
$$

and the relative error as

$$
E_{r}(t)=\frac{\left|q_{s}(t)-q_{c}(t)\right|}{\left|q_{s}(t)\right|}
$$

The exact solution $q_{s}$ of the scattering problem is known analytically only for the case of scattering of a plane wave by a circle. For the other cases, the error is estimated by comparing computed solutions to each other.

The relative and absolute error for the scattering of a plane wave by the circle is shown in Figure 6.4 for increasing values of $k$. We have chosen to use cubic spline basis functions and derivatives up to order $d=1$ in each specialized quadrature rule. The figures show that both the absolute error and the relative error decrease with increasing $k$. This is due to the higher accuracy of the quadrature rule $Q_{c}[f ; t]$ at larger frequencies. The relative error tends to $100 \%$ in the deep shadow region because we have approximated the solution by 0 in that region. One can verify from 


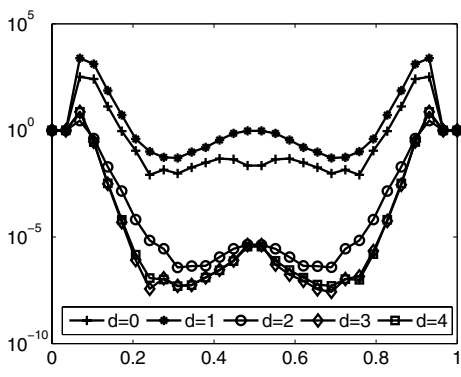

(a) Relative error for the case of a circle

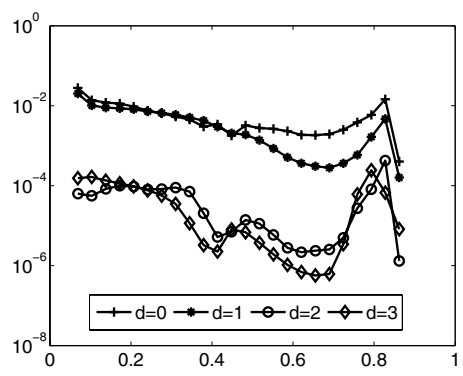

(b) Absolute error for the case of an ellipse

FIG. 6.5. Comparison of the absolute and relative error at $k=200$ for different values of $d$, the number of derivatives used in the specialized quadrature rules. The left panel corresponds to the case of column 1 in 6.1, the right panel corresponds to the case of column 4.

TABLE 6.2

Maximal absolute error as a function of the size of the shadow boundary region, and pointwise absolute and relative error in a fixed point $T=0.13$ in the shadow boundary region. The constants $C_{j}=C$ and $D_{j}=D$ are chosen pairwise equal $\left(k=200, d=2, N_{j}=50\right)$.

\begin{tabular}{lllll}
\hline$(C, D)$ & $(1.0,0.7)$ & $(1.1,0.8)$ & $(1.2,0.9)$ & $(1.3,1.0)$ \\
\hline$\left\|E_{a}\right\|_{\infty}$ & $1.0 E-3$ & $4.6 E-4$ & $2.4 E-4$ & $1.8 E-4$ \\
\hline$E_{a}(T)$ & $4.4 E-4$ & $7.9 E-5$ & $3.6 E-5$ & $5.1 E-6$ \\
$E_{r}(T)$ & $3.2 E-1$ & $5.6 E-2$ & $2.6 E-2$ & $3.7 E-3$ \\
\hline
\end{tabular}

the figures that the absolute error in that region is still quite small compared to the average value of the function $q_{s}(t)$. At $k=1600$, a relative error of order $10^{-5}$ is achieved in the illuminated region and near the shadow boundary.

The accuracy shown in Figure 6.4 for the smaller value $k=200$ is quite poor, however. The accuracy is much improved by increasing the number of derivatives used in the quadrature rules. This is illustrated in Figure 6.5(a) for the same scattering example with $k=200$. The number of derivatives $d$ ranges from 0 to 4 . We used cubic spline basis functions for $d=0,1,2$ and splines of degree 5 for $d=3,4$. A relative error of order $10^{-8}$ is achieved in the shadow boundary region, and a relative error of order $10^{-5}$ in the illuminated region. A similar experiment is illustrated in Figure 6.5(b), corresponding to an incident circular wave on an ellipse-shaped boundary. The error for $d=0, \ldots, 3$ in this case was estimated by comparing to the results of using $d=4$.

The constants $C_{1}, C_{2}, D_{1}$, and $D_{2}$ determine the size of the transitional shadow boundary regions. Since the solution is approximated by zero in the shadow region outside these intervals, it can be expected that increasing the value of these parameters results in smaller errors. This is confirmed by the results in Table 6.2, which show the maximum of the absolute error $E_{a}$. The maximum is reached in the points $t_{s b 1}-$ $D_{1} k^{-1 / 3}$ and $t_{s b 2}+D_{2} k^{-1 / 3}$, i.e., on the border of the shadow region. It is also shown that the accuracy of a point in the shadow boundary region increases if the size of that region is increased. The accuracy of the points in the illuminated region is unaffected by this change.

Finally, Table 6.3 shows the result of increasing the total number of degrees of 
TABLE 6.3

Maximal absolute error as a function of the number of degrees of freedom.

\begin{tabular}{lccccc}
\hline$N_{1}=N_{2}=N_{3}$ & 20 & 30 & 40 & 50 & 60 \\
\hline$\left\|E_{a}\right\|_{\infty}$ & $1.5 E-2$ & $2.4 E-3$ & $4.9 E-4$ & $2.2 E-4$ & $1.9 E-4$ \\
\hline
\end{tabular}

freedom for the same scattering example. The accuracy initially increases rapidly, but remains stable as $N$ increases further.

In conclusion, the accuracy of the proposed method is influenced by a number of factors: the value of $k$, the accuracy of the specialized quadrature rules, the size of the shadow boundary region, and the number of degrees of freedom. The results show that the error is quite small and can be improved by changing the parameters. However, the experiments do not indicate that arbitrary accuracy can be achieved for a given value of $k$. For achieving high accuracy at very low values of $k$, the use of specialized quadrature rules is not recommended; one can instead employ the dense discretization described in section 5.1.

7. Concluding remarks. The method presented in this paper achieves a sparse discretization matrix for an integral equation, which is made possible by the presence of strong oscillations. The method is based on a classical boundary element technique near the shadow boundary, and uses a new quadrature formula that is very effective for oscillatory integrals in the illuminated region. The a priori knowledge of the phase of the solution allows a discretization with a fixed small number of unknowns. Since the new quadrature rule requires only few quadrature points, a sparse discretization matrix is obtained. Moreover, numerical results indicate that the accuracy of the solution increases with increasing frequency.

The method can still be improved in a number of ways. For example, we have only considered a localized Filon-type method, while numerical experiments indicate that regular Filon-type methods are typically more accurate for the same frequency. One could also add additional quadrature points besides the singular and stationary points to improve the accuracy. The small densely discretized part near the shadow boundary may possibly be avoided by using a more elaborate ansatz for the asymptotic behavior of the solution, such as those described by the geometric theory of diffraction $[17,23$, $16]$.

The method is limited to smooth convex obstacles. The approach of [7] for smooth convex obstacles can be extended to multiple scattering configurations using an iterative approach [14]. We expect the same will hold for our approach. The extension to three-dimensional problems is the subject of future research. This might be based on suitable cubature rules for multivariate highly oscillatory integrals which have recently been constructed [18].

\section{REFERENCES}

[1] T. Abboud, J. NÉdÉlec, And B. Zhou, Méthode des équations intégrales pour les hautes fréquences, C. R. Acad. Sci. Paris, 318 (1994), pp. 165-170.

[2] K. R. Aberegg and A. F. Peterson, Application of the integral equation-asymptotic phase method to two-dimensional scattering, IEEE Trans. Antennas Propagat., 43 (1995), pp. $534-537$.

[3] M. Abramowitz and I. A. Stegun, Handbook of Mathematical Functions with Formulas, Graphs, and Mathematical Tables, Dover Publications, New York, 1965.

[4] P. Bettess, Short wave scattering, problems and techniques, Phil. Trans. R. Soc. Lond. A, 362 (2004), pp. 421-443. 
[5] N. Bleistein and R. Handelsman, Asymptotic Expansions of Integrals, Holt, Rinehart and Winston, New York, 1975.

[6] N. Bleistein, Asymptotic expansions of integral transforms of functions with logarithmic singularities, SIAM J. Math. Anal., 8 (1977), pp. 655-672.

[7] O. P. Bruno, C. A. Geuzaine, J. A. Monro, and F. Reitich, Prescribed error tolerances within fixed computational times for scattering problems of arbitrarily high frequency: The convex case, Phil. Trans. R. Soc. Lond. A, 362 (2004), pp. 629-645.

[8] D. Colton And R. Kress, Integral Equation Methods in Scattering Theory, Wiley, New York, 1983.

[9] R. Cools And A. Haegemans, Algorithm 824: CUBPACK: A package for automatic cubature; Framework description, ACM Trans. Math. Softw. 29 (2003), pp. 287-296.

[10] E. DARve, The fast multipole method: Numerical implementation, J. Comput. Phys., 160 (2000), pp. 195-240.

[11] P. J. Davis And P. Rabinowitz, Methods of Numerical Integration, Comput. Sci. Appl. Math., Academic Press, New York, 1984.

[12] V. Domínguez, I. G. Graham, and V. P. Smyshlyaev, A hybrid numerical-asymptotic boundary integral method for high-frequency acoustic scattering, Numer. Math., 106 (2006), pp. $471-510$.

[13] V. A. Fock, Electromagnetic Diffraction and Propagation Problems, Pergamon Press, New York, 1965.

[14] C. Geuzaine, O. Bruno, and F. Reitich, On the $O(1)$ solution of multiple-scattering problems, IEEE Trans. Magn., 41 (2005), pp. 1488-1491.

[15] E. Giladi And J. B. Keller, A hybrid numerical asymptotic method for scattering problems, J. Comput. Phys., 174 (2001), pp. 226-247.

[16] E. GILADI, Asymptotically derived boundary elements for the Helmholtz equation in high frequencies, J. Comput. Appl. Math., 198 (2007), pp. 52-74.

[17] R. F. Harrington, Time-Harmonic Electromagnetic Fields, McGraw-Hill, New York, 1961.

[18] D. Huybrechs and S. Vandewalle, The construction of cubature rules for multivariate highly oscillatory integrals, Math. Comp., (2007), to appear.

[19] D. Huybrechs and S. Vandewalle, On the evaluation of highly oscillatory integrals by analytic continuation, SIAM J. Numer. Anal., 44 (2006), pp. 1026-1048.

[20] A. Iserles, S. P. Nørsett, And S. Olver, Highly oscillatory quadrature: The story so far, in Proceedings of ENuMath 2005, Santiago de Compostela, Berlin, 2006, Springer-Verlag, New York, 2006, pp. 97-118.

[21] A. IserLes AND S. P. NøRSETt, Efficient quadrature of highly oscillatory integrals using derivatives, Proc. R. Soc. Lond. Ser. A Math. Phys. Eng. Sci., 461 (2005), pp. 1383-1399.

[22] D. S. Jones, High-frequency refraction and diffraction in general media, Phil. Trans. R. Soc. Lond. A, 255 (1963), pp. 341-387.

[23] J. B. Keller, Geometrical theory of diffraction, J. Opt. Soc. Amer., 52 (1962), pp. 116-130.

[24] S. Langdon And S. N. Chandler-Wilde, Implementation of a boundary element method for high frequency scattering by convex polygons, in Proceedings of the 5th U.K. Conference on Boundary Integral Methods, K. Chen, ed., 2005, University of Liverpool, Liverpool, UK, 2005 , pp. $2-11$.

[25] S. Langdon and S. N. Chandler-Wilde, A wavenumber independent boundary element method for an acoustic scattering problem, SIAM J. Numer. Anal., 43 (2006), pp. 24502477.

[26] D. Levin, Fast integration of rapidly oscillatory functions, J. Comput. Appl. Math., 67 (1996), pp. 95-101.

[27] R. B. Melrose And M. E. TAYLOR, Near peak scattering and the corrected Kirchhoff approximation for a convex obstacle, Adv. in Math., 55 (1985), pp. 242-315.

[28] J.-C. NÉDÉLEC, Acoustic and Electromagnetic Equations, Appl. Math. Sci. 144, Springer, Berlin, 2001.

[29] S. Olver, Moment-free numerical integration of highly oscillatory functions, IMA J. Numer. Anal., 26 (2006), pp. 213-227.

[30] V. Rokhlin, Diagonal forms of translation operators for the Helmholtz equation in three dimensions, Appl. Comput. Harmon. Anal., 1 (1993), pp. 82-93.

[31] E. M. Stein, Harmonic Analysis: Real-Variable Methods, Orthogonality and Oscillatory Integrals, Princeton University Press, Princeton, NJ, 1993.

[32] G. N. Watson, Harmonic functions associated with the parabolic cylinder, Proc. London Math. Soc. Ser. 2, 17 (1918), pp. 116-148.

[33] R. Wong, Asymptotic Approximation of Integrals, Classics in Appl. Math. 34, SIAM, Philadelphia, 2001. 